\title{
Correlation between Family $R B 1$ Gene Pathogenic Variant with Clinical Features and Prognosis of Retinoblastoma under 5 Years Old
}

\author{
Yi Zhang, ${ }^{1}$ Yizhuo Wang, ${ }^{1}$ Dongsheng Huang $\left(\mathbb{D},{ }^{1}\right.$ Jianmin $\mathrm{Ma},{ }^{2}$ Weiling Zhang, ${ }^{1}$ Huali Gu, \\ Yan Zhou, ${ }^{1}$ You Yi, ${ }^{1}$ and Pinwei Zhang ${ }^{1}$ \\ ${ }^{1}$ Department of Pediatrics, Beijing Tongren Hospital, Capital Medical University, No 2, Xihuan South Road, Yizhuang Economic and \\ Technological Development Zone, Beijing 100176, China \\ ${ }^{2}$ Beijing Tongren Eye Center, Beijing Tongren Hospital, Capital Medical University, Beijing Institute of Ophthalmology, Beijing Key \\ Laboratory of Ophthalmology and Visual Sciences, Beijing 100730, China
}

Correspondence should be addressed to Dongsheng Huang; dongshenghuang99@163.com

Received 4 March 2021; Revised 14 June 2021; Accepted 3 July 2021; Published 13 July 2021

Academic Editor: Giulia Bivona

Copyright ( $) 2021$ Yi Zhang et al. This is an open access article distributed under the Creative Commons Attribution License, which permits unrestricted use, distribution, and reproduction in any medium, provided the original work is properly cited.

\begin{abstract}
Retinoblastoma (RB) is the most common primary intraocular malignant tumor in infants and the prototype of human hereditary tumors. Its occurrence and development are closely related to the pathogenic variant of tumor suppressor RB1 gene. We aim to analyze the characteristics of RB1 gene pathogenic variant and clinical phenotype in retinoblastoma patients and their relatives. Children with RB were recruited from August 2007 to November 2017. QT-PCR, probing, and gene sequencing were used to analyze the sequence of $R B 1$ gene in RB children, their parents, or grandparents with a clear history of illness. The SPSS20.0 software was used to analyze the correlation between polymorphisms of $R B 1$ gene and the incidence and prognosis of the enrolled children and relatives. $40 \mathrm{RB}$ children (20 males and 20 females) were recruited, unilateral RB accounted for $52.5 \%$ (21/40), bilateral RB accounted for 42.5\% (17/40), and trilateral RB accounted for 5.0\% (2/40). 6 patients had a clear family history $(15.0 \%, 6 / 40)$. It had been verified that 19 probands $(47.5 \%)$ have $R B 1$ gene pathogenic variants (11 frameshift and 8 missense pathogenic variants), of which germline inheritance accounted for $47.4 \%$ (9/19) and nongermline heredity accounted for $52.6 \%$ (10/19). Pathogenic variants of 10 nucleic acid sites without reported were found, among which c.2455C $>$ G (p.L819V) was confirmed to have heterozygous pathogenic variants in both a bilateral RB patient and his mother with unilateral RB. Family genetic high-risk factors, bilateral/trilateral $\mathrm{RB},>12$-month-onset $\mathrm{RB}$ have a higher proportion of $R B 1$ gene pathogenic variant than children with no family history, unilateral $\mathrm{RB}$, and $\leq 12$-month $(P=0.021,0.001,0.034)$. The proportion of pedigree inheritance of infantile retinoblastoma with bilateral disease is high. There was a certain proportion of $R B 1$ gene pathogenic variant in 3-5-year-old children with bilateral $\mathrm{RB}$, even if they had no family genetic history. Therefore, the detection of $R B 1$ gene pathogenic variant should not only focus on infants but also on the phenotype of $R B 1$ gene pathogenic variant in children over 3 years old with bilateral eye disease.
\end{abstract}

\section{Introduction}

Retinoblastoma (RB) is the most common primary intraocular malignant tumor in infants and the prototype of human hereditary tumors. RB could be divided into two genetic patterns of germline inheritance: germline inheritance and nongermline inheritance [1]. The germline inheritance is mainly germ cell pathogenic variant, presenting with bilateral RB (account for about 20-30\%) or unilateral multiple RB (account for about $70-80 \%$ ), of which about $5 \%$ can be accompanied by intracranial tumors, such as pineal gland tumor and primary neuroblastoma on or near the sella turcica, which is called trilateral RB [2]. Nongermline inheritance is caused by somatic pathogenic variant, mostly unilateral $\mathrm{Rb}$. Of all $\mathrm{RB}$, genetic $\mathrm{RB}$ account for about $45 \%$, nongenetic RB account for 55\%, and about 5\%-10\% of 
children have a clear family history $[1,3-6]$. The occurrence and development of $\mathrm{RB}$ are closely related to the pathogenic variant of the tumor suppressor RB1 gene [7]. Since the 1980s, the $R B 1$ gene on the long arm of chromosome 13 (13q14) has been recognized as the RB tumor suppressor gene for the first time, and its pathogenic variant is involved in the occurrence of $\mathrm{RB}[5,6,8-10]$. Compelling studies $[1,5,6,11-15]$ indicated that the $R B 1$ gene is an allele encoding retinoblastoma protein (pRB), and the lack of monitoring or inactivation of $R B 1$ will lead to a decline in cell proliferation regulation function thus resulting in abnormal cell proliferation.

At present, the use of PCR, first-generation sequencing technology, high-throughput sequencing, and probe technology could detect $R B 1$ gene pathogenic variant, which provides a useful reference for prognosis evaluation, treatment planning, and family planning consultation [5, 12-14]. However, gene pathogenic variant often results in the failure of protein translation and synthesis due to the nucleic acid or base pathogenic variant in the coding region involved in protein translation and transcription. The detection of chromosome 13 and the pathogenic variant of the $R B 1$ gene are well reported [5], but the mechanism of the analysis of the nucleic acid polymorphism of the coding region on the pathogenesis and efficacy of RB is relatively rare.

Since 2005, our hospital has collected more than 2,000 retinoblastoma cases; however, the proportion of children with clear family history is less than $3 \%$, and some children with unilateral eye disease appeared heterogeneous binocular disease. The detection and analysis of $R B 1$ gene pathogenic variant may be helpful for the early development and early warning of disease progression. Due to the late application of genetic technology, the study on the relation between clinical therapy and the detection of polymorphisms in the familial $R B 1$ gene is rare. In this study, we collected 40 cases of pathological/clinically diagnosed children with RB. The RB1 gene polymorphism was measured in these children and their parents. For children with a clear family history, their maternal grandparents were simultaneously analyzed with informed consent. Combined with their clinical data, we analyze the impact of $R B 1$ gene polymorphism on morbidity of $\mathrm{RB}$ and provide a molecular level diagnosis information.

\section{Patients and Methods}

2.1. Patients. In this retrospective analysis, the diagnosis and treatment procedure have been approved by the ethics committee (TRECKY2019-034). From August 2007 to November 2017, a series of cases were included. The informed consent was obtained from the legal guardian of the patients. The inclusion criteria were as follows: (1) the sample is collected before the chemotherapy treatment, (2) male or female patients with the first onset age of $<5$ years ( 60 months), (3) RB was initial diagnosed histopathological and initial treated in our hospital, and (4) complete follow-up data and informed consent with regular treatment and followup. Rejection or withdrawal criteria: patients who did not meet one of the above criteria are rejected or withdrawn.
2.2. Diagnostic Criteria [16-18]. The clinical diagnosis was based on transocular ultrasonography, and Retcam machine fundus examination and orbital CT/MRI suggesting that the occupancy of the lesion is within the sphere and calcification is visible. The diagnosis was confirmed to be consistent with the diagnosis of RB by histopathology and immunohistochemistry. According to the international classification of retinoblastoma (ICRB) in 2003 [19], RB was classified into I-IV stages. According to the IIRC system, the intraocular stage was divided into A-E groups for the serious classification. Other imaging examinations including fundus examination under general anesthesia by Retcam, whole-body bone scan combined chest and abdominal enhanced pelvis CT. Patients were divided into 4 groups according to risk factors: low-risk group (no high-risk factors), intermedium-risk group (with 1 high-risk factor), high-risk group (infringement of optic nerve ends, or 2 or more high-risk factors), and ultrahigh-risk group (with intracranial metastasis or distant metastasis).

2.3. Samples. A total of $5 \mathrm{ml}$ whole blood samples were drawn from each child into EDTA tubes before treatment. $10 \mathrm{ml}$ of whole blood from the relatives of the child was taken on fasting. Phenol chloroform method was used to extract the whole DNA (QLA DNA blood mini kit, Qiagen). First, add 3-5 ml of whole blood into a $50 \mathrm{ml}$ centrifuge tube; add $30-40 \mathrm{ml}$ of $\mathrm{ddH}_{2} \mathrm{O}$; shake for 20 seconds; stand for 10 minutes, 4 degrees, $3800 \mathrm{rpm}, 20 \mathrm{~min}$; and remove the supernatant carefully. Next, $5 \mathrm{ml}$ TES were added to the precipitation to mix it upside down (vortex oscillation, breaking cell membrane). Then, $350 \mu \mathrm{l}, 10 \% \mathrm{SDS}$, and $70 \mu \mathrm{l}(10 \mathrm{ng} / \mu \mathrm{l})$ proteinase $\mathrm{K}$ were added at $37^{\circ} \mathrm{C}$ overnight. The next day, the centrifuge tube was cooled to room temperature, $5 \mathrm{ml}$ of Trissaturated phenol was first added and mixed upside down. After that, $5 \mathrm{ml}$ of isoamyl chloroform $(24: 1)$ was added (must be done, otherwise, it will affect the final stratification and is not conducive to the extraction of supernatant), followed by upside down mixing, 4 degrees, $2500 \mathrm{rpm}$, and centrifugation for $15 \mathrm{~min}$. Take all the supernatant into another centrifuge tube, add 2.5 times volume of ice-free ethanol, repeatedly reverse, precipitate out the DNA blob directly into the $1.5 \mathrm{ml}$ centrifuge tube with pipette, wash it twice with $75 \%$ ethanol, and add $200 \mu \mathrm{l}$ or $300 \mu \mathrm{l}$ after drying dissolve $\mathrm{TB}$, put it $4^{\circ} \mathrm{C}$ later to measure $\mathrm{OD}$, place the original solution at $-20^{\circ} \mathrm{C}$ or $-80^{\circ} \mathrm{C}$ for storage. The concentration of the extracted DNA sample was $20 \mathrm{ng} / \mathrm{dl}$. The primers and sequence analysis of the $R B 1$ gene for amplification were designed by Joy Orient Translational Medicine Research Center Co., Ltd. China. Primer sequence: F-3' -GTAACCAGGTC ATGTAGCA-5' ${ }^{\prime}$ R-3' -GCCTTAGGTAGACGGATC-5'.

2.4. High-Throughput Sequencing of RB1 Gene. Online database (https://www.ncbi.nlm.nih.gov/assembly/CF_ $000001405.39 /)$ indicates the total length of the RB1 gene (13q14.2) sequence is 3,099,706,404 bp, 28 exons, and some adjacent introns. A nucleic acid analysis (Agilent Sureselect Target Enrichment human-exome system) was used for $R B 1$ gene analysis. The DNA sequence analysis is based on the GATK standard establishment algorithm (GATK V.4, 


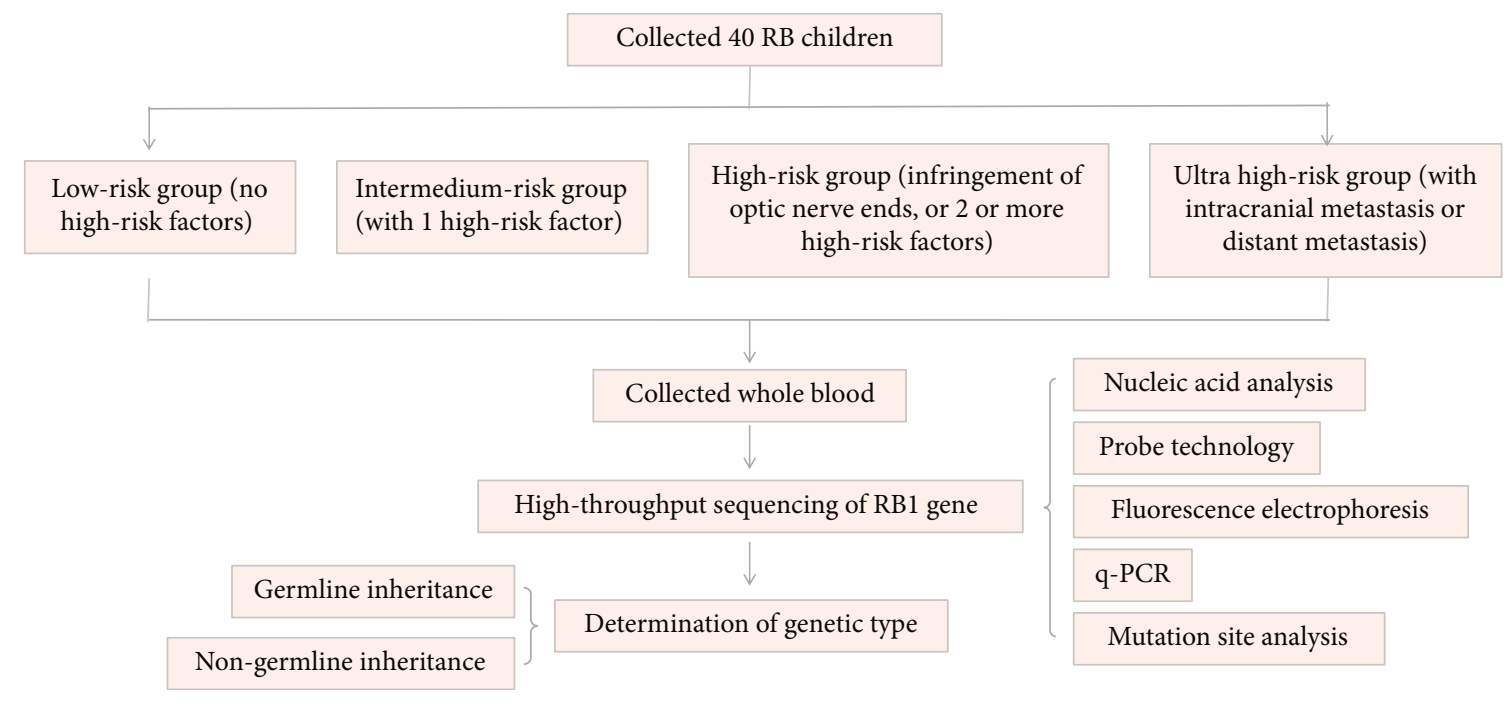

FIgURE 1: Schematic representation of the study.

version 2.0). To detect the pathogenic variants, deletions, and insertions of nucleic acid sites in the $R B 1$ gene, probe technology, fluorescence electrophoresis, QT-PCR (Applied Biosystems Foster City, CA, USA), and fragment sequencing were used for verification. All mutation sites will be checked online based on 1000 Genomes data (http://http://www.1000 .genomes.org/), nucleic acid, and base mutations (http:// www.ncbi.nih.gov/projects/snp/). Mutation on exons was represented by c.-xxx, and introns are represented by IVS $+\mathrm{xxx}$. Pathogenic variant type includes base insertion (Ins) and deletion (del). The base length unit is bp. Undefined germline genetic pathogenic variant of $R B 1$ gene refers to the frameshift or missense pathogenic variant of nucleic acid or base point in the coding region of $R B 1$ gene, but it has not been confirmed by online database or domestic and foreign harmful pathogenic variants.

2.5. Determination of Genetic Type [1, 4-6]. (1) Germline inheritance could be confirmed if direct family members clearly have $\mathrm{RB}$ patients with or without $R B 1$ locus variation; $R B 1$ gene has locus variation, and the same locus variation exists in relatives. (2) Nongermline inheritance: only the proband's $R B 1$ gene site pathogenic variant exists, and there are no high-risk relatives and genetic site pathogenic variants

2.6. Statistical Analysis. The SPSS 20.0 software was used for analysis. Statistical description and frequency description were used for quantitative data. The measurement data of normal distribution is (average \pm standard deviation), and that of nonnormal distribution is M (Q1, Q3). The count data is expressed as the composition ratio or percentage (\%). Kaplan Meier curve was used for survival analysis, and Cox regression was used to analyze the influence of risk factors on prognosis. Fisher's precise inspection was used for statistical analysis. $P<0.05$ was considered as a statistical difference.

\section{Results}

3.1. Demographic Data. 40 patients were enrolled with 20 males and 20 females. Monocular disease occurred in 21 cases $(52.5 \%)$ and 17 cases $(42.5 \%)$ with bilateral eyes. The other 2 cases (5.0\%) with trilateral RB and the number of disease eyes were 61 . The median age of these children was 15.5 months (1-53 months) (Figure 1 and Table 1). Among the 40 children, 6 had a family history (15.0\%), and 34 had no clear family history (85.0\%). After diagnosis, ophthalmectomy (removal of heavy eyes in children with two eyes) was performed in 23 cases, and 2 cases of RB patients with trilateral eyes were classified as grade IV due to pineal gland occupancy. The composition of pathology grade was grade I accounted for $48 \%(12 / 25)$, grade II accounts for $16.0 \%$ (4/25), grade III accounts for $24.0 \%(6 / 25)$, and grade IV accounts for $12.0 \%(3 / 25)$. Vitrectomy was performed in 2 cases; 3 patients received interventional therapy; 6 patients underwent sheath injection according to pathological grading. 39 patients received chemotherapy. The median chemotherapy cycle was 6 cycles (2-17 cycles).

3.2. RB1 Gene Analysis. Mutations, insertions, deletions, and other site pathogenic variants of $R B 1$ gene occurred in 19 of 40 patients $(47.5 \%)$, of which 11 were frameshift pathogenic variants $(57.9 \%, 11 / 19)$, and of which 8 were inserted missense pathogenic variants $(42.1 \%, 8 / 19)$; no pathogenic variants were found in 21 cases of children (52.5\%). Combined with family history and $R B 1$ gene phenotype, 9 cases of RB were germline inheritance in genetic type $(22.5 \%, 9 / 40), 10$ cases were RB1 gene site pathogenic variants $(25.0 \%$, $10 / 40$ ), and the remaining 21 cases had no evidence of genetic tendency $(52.5 \%, 21 / 40)$. Fisher's clinical analysis suggested that for children with germline inheritance undergo eyeball removal, the ratio of postoperative tumor invasion to the posterior bulb optic nerve/end was higher than that of children with only tumor gene site pathogenic variants $(P=0.025)$. Among the 9 cases of germline 
Table 1: Demographic data of the 40 children.

\begin{tabular}{|c|c|}
\hline Category & $N(\%)$ \\
\hline Age & 40 \\
\hline $0 \sim 12$ months & $13(32.5 \%)$ \\
\hline$>12$ months & $27(67.5 \%)$ \\
\hline $12-36$ months & $23(85.2 \%)$ \\
\hline $36-60$ months & $4(14.8 \%)$ \\
\hline Family genetic history & 40 \\
\hline With family history & $6(15 \%)$ \\
\hline Bilateral RB & 5 \\
\hline Trilateral RB & 1 \\
\hline Without family history & $34(85 \%)$ \\
\hline Unilateral RB & 21 \\
\hline Bilateral RB & 12 \\
\hline Trilateral RB & 1 \\
\hline Treatment & 40 \\
\hline $\begin{array}{l}\text { Interventional therapy+chemotherapy+intrathecal } \\
\text { injection }\end{array}$ & $13(32.5 \%)$ \\
\hline Chemotherapy \pm intrathecal injection & $2(5 \%)$ \\
\hline Eyeball removal+chemotherapy \pm intrathecal injection & $22(55 \%)$ \\
\hline $\begin{array}{l}\text { Eyeball removal+vitrectomy+chemotherapy } \\
\pm \text { interventional therapy } \pm \text { intrathecal injection }\end{array}$ & $1(2.5 \%)$ \\
\hline Chemotherapy+vitrectomy & $1(2.5 \%)$ \\
\hline Risk grouping & 40 \\
\hline LR & $29(72.5 \%)$ \\
\hline IR & $6(15.0 \%)$ \\
\hline HR & $5(12.5 \%)$ \\
\hline Prognosis & 40 \\
\hline Survival & $31(77.5 \%)$ \\
\hline Dead & $6(15.0 \%)$ \\
\hline Lost follow-up & $3(7.5 \%)$ \\
\hline Onset eye and genetic type & 40 \\
\hline Unilateral RB & 21 \\
\hline $\begin{array}{l}\text { Germline inheritance/tumor gene pathogenic } \\
\text { variant/no genetic characteristics }\end{array}$ & $2 / 3 / 16$ \\
\hline Bilateral RB & 17 \\
\hline $\begin{array}{l}\text { Germline inheritance/tumor gene pathogenic } \\
\text { variant/no genetic characteristics }\end{array}$ & $6 / 6 / 5$ \\
\hline Trilateral RB & 2 \\
\hline Germline inheritance/tumor gene pathogenic variant & $1 / 1$ \\
\hline Genotype & 19 \\
\hline Germline inheritance* & 9 \\
\hline $\begin{array}{l}\text { Frameshift pathogenic variant/base deletion } \\
\text { or insertion/no pathogenic variant }\end{array}$ & $7 / 1 / 1$ \\
\hline Nongermline inheritance & 10 \\
\hline $\begin{array}{l}\text { Frameshift pathogenic variant/base deletion } \\
\text { or insertion }\end{array}$ & $4 / 6$ \\
\hline
\end{tabular}

${ }^{*} 1$ case with a clear family history proband and diseased relative (mother) RB1 gene has no harmful pathogenic variants; ", no children underwent binocular removal. inheritance, 6 cases have a clear family history. Three children and relatives have the same pathogenic variant site. 21 children and their relatives had no pathogenic variant phenotype (52.5\%), of which 1 patient with binocular disease had a family genetic history, 1 patient had congenital motor dysplasia, and the remaining 19 patients had no family genetic and high-risk factors for congenital diseases (Tables 1 and 2). We found 10 probands with novel genetic variants: c.2124_2125insT, c.2455C > G, IVS1+372G $>C$, c.1686_1688delATG, c.2092A>T, IVS18+1_47del47, c.376_ 380; IVS3+1_25delinsGGAAACGAATT, c.425_428delCCAA, c.62delC, IVS4+50T>C, Table 3.

As we can see from Table 3 (case 2), the proband and the mother of the proband simultaneously had a heterozygous pathogenic variant at position c.2455C $>\mathrm{G}$ (p.L819V). In Table 3 (case 8 ), the proband had a missense pathogenic variant in base deletion. Although the RB1 gene pathogenic variant was not detected in relatives, the mother was a patient. The proportion of $R B 1$ gene site pathogenic variant is higher in those with family genetic high-risk factors, binocular/trilateral $\mathrm{RB},>12$-month-onset $\mathrm{RB}$, and in the high-risk group than those with no family history, unilateral eye $R B, \leq 12$ month-old and low-risk group $(P=0.021,0.001,0.034$, 0.049 , respectively) Table 4 . The pathogenic variant was correlated with pathological grade after eyeball removal with significant difference $(R=4.753, P=0.029)$.

3.3. RB1 Gene Pathogenic Variants and Diagnosis of Age Feature. In $13 \mathrm{RB}$ cases aged $<12$ months old, only 4 bilateral $\mathrm{RB}$ cases had $R B 1$ gene pathogenic variant (one of frameshift pathogenic variants, two of excluding deletion or loss of heterozygosity). According to family history and kindred phenotype, the genetic type of 3 bilateral RB was germline inheritance, and only one bilateral RB patient's genetic type was gene site mutation. The difference of pathogenic variant type in infant bilateral RB cases was significant $(P=0.049)$ (Tables 5 and 6). Other 4 bilateral RB and 6 unilateral RB had no $R B 1$ gene pathogenic variant. Although the proportion of $R B 1$ gene pathogenic variant in bilateral disease was higher than unilateral cases, statistical analysis showed that there was no significant difference in $R B 1$ gene pathogenic variant between $<12$ months old children $(P=0.122)$ (Tables 5 and 6).

In $27 \mathrm{RB}$ cases aged $>12$ months old ( 23 cases were 12 36 months old and 4 cases were 36-60 months old), RB1 gene pathogenic variants were found in 16 of 27 children (59.3\%), including 10 of frameshift pathogenic variants or excluding deletion or loss of heterozygosity $(62.5 \%, 10 / 16)$, and 6 cases were germline inheritance ( 2 unilateral $\mathrm{RB}$ cases and 4 bilateral/trilateral RB cases) $(37.5 \%, 6 / 16)$, as shown in Tables 6 and 7. Statistical analysis showed that the proportion of $R B 1$ gene pathogenic variant in bilateral and/or trilateral $\mathrm{RB}$ was significantly higher than that of unilateral $\mathrm{RB}$ $(P=0.003)$ (Table 5). In 23 patients aged 12-36 months old, the proportion of $R B 1$ gene pathogenic variants in bilateral eyes/trilateral eyes $(88.9 \%, 8 / 9)$ was higher than that in unilateral eyes $(25.7 \%, 5 / 14)$. The pathogenic variant type was mainly $R B 1$ gene site mutation. The differences were all significant $(P=0.027,0.025)$ (Tables 6 and 7$)$. In 4 RB 


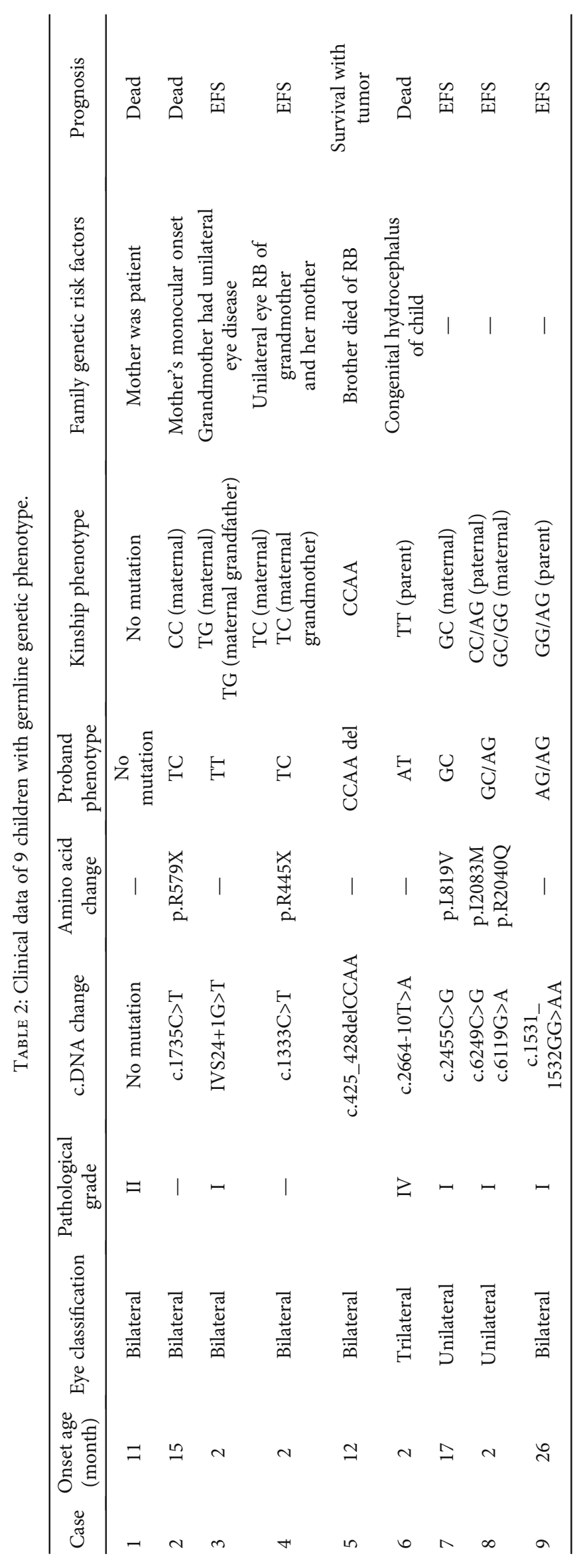




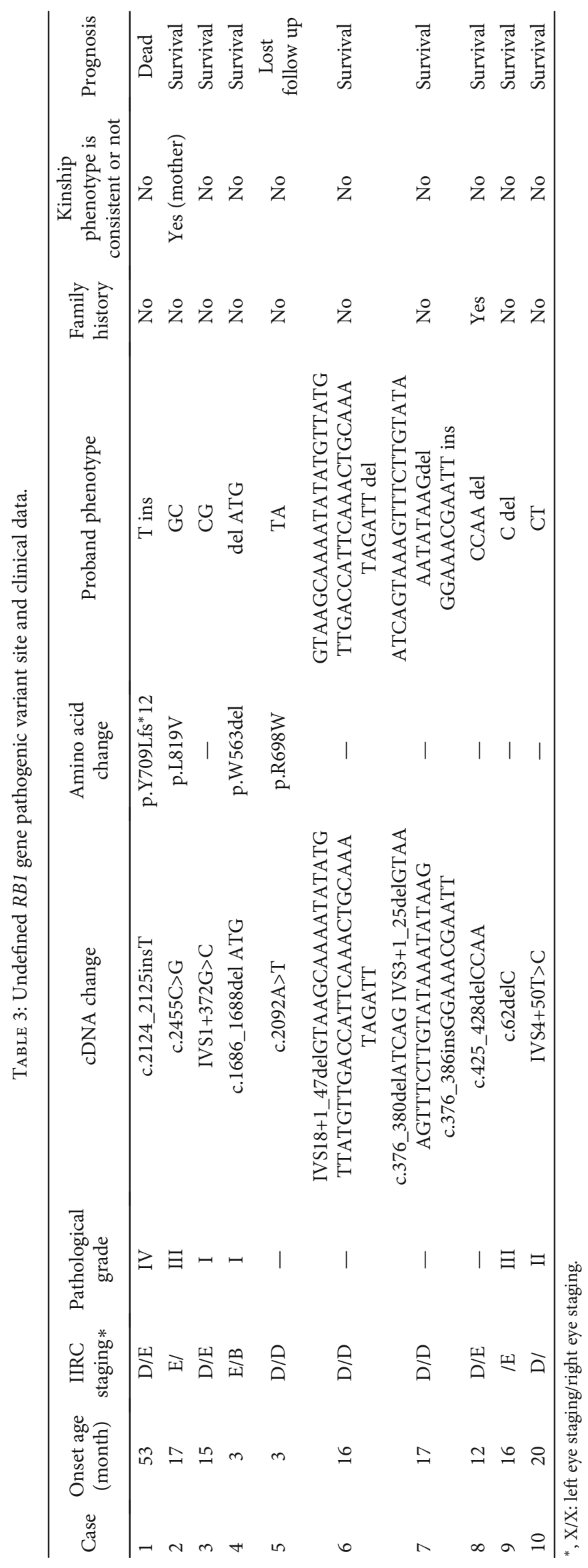


TABLE 4: Analysis of $R B 1$ gene pathogenic variants and onset characteristics.

\begin{tabular}{|c|c|c|c|c|c|}
\hline Onset characteristics & $N$ & $\begin{array}{l}R B 1 \text { gene pathogenic } \\
\text { variant cases }(\mathrm{m})\end{array}$ & $\%(\mathrm{~m} / \mathrm{n})$ & $\begin{array}{c}\text { Statistics } \\
\text { value }\left(X^{2}\right)\end{array}$ & $P$ \\
\hline Age (mon) & 40 & 19 & $47.5(19 / 40)$ & \multirow{4}{*}{4.607} & \multirow{4}{*}{0.019} \\
\hline $0 \sim 12$ & 13 & 3 & $18.6(3 / 13)$ & & \\
\hline $12-36$ & 23 & 13 & $56.5(13 / 23)$ & & \\
\hline 4 & 4 & 3 & $75.0(3 / 4)$ & & \\
\hline Eye classification & 40 & 19 & $47.5(19 / 40)$ & \multirow{3}{*}{10.119} & \multirow{3}{*}{0.002} \\
\hline Unilateral RB & 21 & 5 & $23.8(5 / 21)$ & & \\
\hline Bilateral RB/trilaterall RB* & 19 & 14 & $76.2(14 / 17)$ & & \\
\hline Risk group & 40 & 19 & $47.5(19 / 40)$ & \multirow{4}{*}{6.278} & \multirow{4}{*}{0.049} \\
\hline Low-risk (LR) group & 29 & 12 & $41.4(12 / 29)$ & & \\
\hline Intermediate-risk (IR) group & 6 & 2 & $33.3(2 / 6)$ & & \\
\hline High-risk group (HR) & 5 & 5 & $100.0(5 / 5)$ & & \\
\hline Family genetic high-risk factors & 40 & 19 & $47.5(19 / 40)$ & \multirow{3}{*}{5.647} & \multirow{3}{*}{0.021} \\
\hline Yes & 10 & 8 & $80.0(8 / 10)$ & & \\
\hline No & 30 & 11 & $36.7(11 / 30)$ & & \\
\hline Congenital developmental defects & 40 & 19 & $47.5(19 / 40)$ & \multirow{3}{*}{0.005} & \multirow{3}{*}{0.731} \\
\hline Yes & 2 & 1 & $50.0(1 / 2)$ & & \\
\hline No & 38 & 18 & $47.4(18 / 38)$ & & \\
\hline Pathology grade of children with eye removal & 23 & 9 & $39.1(9 / 23)$ & \multirow{3}{*}{0.059} & \multirow{3}{*}{0.582} \\
\hline Posterior optic nerve un infringed & 16 & 6 & $37.5(6 / 16)$ & & \\
\hline Invasion of the posterior optic nerve and end of bulb & 7 & 3 & $14.3(3 / 7)$ & & \\
\hline
\end{tabular}

${ }^{*}: 2$ of 40 cases were trilateral RB and all had RB1 gene pathogenic variant.

TABLE 5: RB1 gene pathogenic variants and diagnosis of age feature in $40 \mathrm{RB}$ patients.

\begin{tabular}{lccccr}
\hline \multirow{2}{*}{ Age } & \multirow{2}{*}{$\mathrm{N}$} & \multicolumn{2}{c}{$R B 1$ gene pathogenic variant } & \multicolumn{2}{c}{ Non-RB1 gene pathogenic variant } \\
& & Unilateral RB $(\% \mathrm{~m} / \mathrm{n})$ & Bilateral RB $(\% \mathrm{~m} / \mathrm{n})$ & Unilateral RB $(\% \mathrm{~m} / \mathrm{n})$ & Bilateral RB $(\% \mathrm{~m} / \mathrm{n})$ \\
\hline$<12$ months & 13 & $0 \%(0 / 13)$ & $23.1 \%(3 / 13)$ & $46.1 \%(6 / 13)$ & $30.8 \%(4 / 13)$ \\
$>12$ months & 27 & $18.5 \%(5 / 27)$ & $40.7 \%(11 / 27)^{*}$ & $37.0 \%(10 / 27)$ & 0.122 \\
\hline
\end{tabular}

*: two patients were trilateral RB.

patients aged 36-60 months old, 2 bilateral RB cases and 1 trilateral RB case had gene mutation, and only 1 case of unilateral eye RB had no gene mutation.

\section{Discussion}

Approximately 9,000 newborn babies are diagnosed with $\mathrm{RB}$ every year. Most of RB onset within 3 years of age without gender differences, while about 3000 children die of RB every year $[20,21]$. Canada reported that the average age of diagnosis of monocular onset is 27 months, the average age of diagnosis of binocular onset is 15 months, and the average age of diagnosis of single and double eyes in Kenya is 36 months and 25 months $[1,3,6]$. In our retrospective study, there were 20 males and 20 females, and RB onset within 5 years of age, with a median age of 15.5 months, which basically consistent with the literature reports $[1,3,22]$.

$\mathrm{RB}$ cells are derived from susceptible vertebral photoreceptor cells of retinal precursors. When alleles of the $R B 1$ gene are mutated or deleted, RB cells could remain in the inner core layer, initially act as benign precursor "retinoma," with gene pathogenic variant increased, aggregation and uncontrolled cell proliferation lead to retinoblastoma [15, 16]. At present, RB gene detection is widely used in screening and detection of RB1 mutation carriers in patients' relatives, as well as prenatal testing [23]. In this study, $47.5 \%$ (19/40) of children with $\mathrm{RB}$ had $R B 1$ gene pathogenic variants, of which $22.5 \%$ (9/40) had germline inheritance (6 cases had a clear family history of onset, 3 cases children and relatives had the same mutated and displayed locus), which is basically consistent with the above report. It is worth noting that one patient and his mother are both RB patients, and the child is binocular and the mother is monocular RB. However, the $R B 1$ gene test showed no harmful mutations, indicating that even without genetic mutations, family genetic risk is still one of the characteristics of germline inheritance, and the pathogenesis of molecular biology should be further studied.

In this group, the incidence of germline genetic and tumor gene pathogenic variant was higher in binocular $\mathrm{RB}$ with significant difference $(P=0.002)$. In this group of cases, 
TABLE 6: RB1 gene pathogenic variants and diagnosis of age and onset eye features in $40 \mathrm{RB}$ patients.

\begin{tabular}{lccc}
\hline Age group (months) & Number $(\% \mathrm{~m} / \mathrm{n})$ & $\begin{array}{c}\text { RB1 gene pathogenic } \\
\text { variant }(\% \mathrm{~m} / \mathrm{n})\end{array}$ & $\begin{array}{c}\text { Non-RB1 gene pathogenic } \\
\text { variant }(\% \mathrm{~m} / \mathrm{n})\end{array}$ \\
\hline 0-12 m & $13(32.5 \% 13 / 40)$ & $23.1(3 / 13)$ & $76.9(10 / 13)$ \\
Unilateral RB & $6(46.2 \% 6 / 13)$ & $0.0(0 / 6)$ & $100.0(6 / 6)$ \\
Bilateral/trilateral RB & $7(46.13 \% 7 / 13)$ & $42.9(3 / 7)$ & $57.1(4 / 7)$ \\
12-36 m & $23(57.5 \% 13 / 40)$ & $56.5(13 / 23)$ & $43.5(10 / 23)$ \\
Unilateral RB & $14(60.9 \% 14 / 23)$ & $35.7(5 / 14)$ & $64.3(9 / 14)$ \\
Bilateral/trilateral RB & $9(39.1 \% 9 / 23)$ & $88.9(8 / 9)$ & $11.1(1 / 9)$ \\
36-60 m & $4(10.0 \% 4 / 40)$ & $75.0(3 / 4)$ & $25.0(1 / 4)$ \\
Unilateral RB & $1(25.0 \% 1 / 4)$ & $0.0(0 / 1)$ & $100.0(1 / 1)$ \\
Bilateral/trilateral RB & $3(75.0 \% 3 / 4)$ & $100.0(3 / 3)$ & $0.0(0 / 3)$ \\
\hline
\end{tabular}

TABLE 7: Types and RB1 genetic analysis of diagnosis of age and onset eye features in 40 RB patients.

\begin{tabular}{lcccc}
\hline Age group (months) & Number $(\% \mathrm{~m} / \mathrm{n})$ & $\begin{array}{c}\text { Germline inheritance } \\
(\% \mathrm{~m} / \mathrm{n})\end{array}$ & $\begin{array}{c}\text { RB1 gene pathogenic } \\
\text { variant }(\% \mathrm{~m} / \mathrm{n})\end{array}$ & $\begin{array}{c}\text { Non-RB1 gene pathogenic } \\
\text { variant }(\% \mathrm{~m} / \mathrm{n})\end{array}$ \\
\hline 0-12 m & $13(32.5 \% 13 / 40)$ & $23.1(3 / 13)$ & $7.7(1 / 13)$ & $69.2(9 / 13)$ \\
Unilateral RB & $6(46.2 \% 6 / 13)$ & $0.0(0 / 6)$ & $0.0(0 / 6)$ & $100.0(6 / 6)$ \\
Bilateral/trilateral RB & $7(46.13 \% 7 / 13)$ & $42.9(3 / 7)$ & $14.3(1 / 7)$ & $42.8(3 / 7)$ \\
12-36 m & $23(57.5 \% 13 / 40)$ & $26.1(6 / 23)$ & $30.4(7 / 23)$ & $43.5(10 / 23)$ \\
Unilateral RB & $14(60.9 \% 14 / 23)$ & $14.3(2 / 14)$ & $21.4(3 / 14)$ & $64.3(9 / 14)$ \\
Bilateral/trilateral RB & $9(39.1 \% 9 / 23)$ & $44.4(4 / 9)$ & $44.4(4 / 9)$ & $11.2(1 / 9)$ \\
36-60 m & $4(10.0 \% 4 / 40)$ & $0.0(0 / 4)$ & $75.0(3 / 4)$ & $25.0(1 / 4)$ \\
Unilateral RB & $1(25.0 \% 1 / 4)$ & $0.0(0 / 1)$ & $0.0(0 / 1)$ & $100.0(1 / 1)$ \\
Bilateral/trilateral RB & $3(75.0 \% 3 / 4)$ & $0.0(0 / 3)$ & $100.0(3 / 3)$ & 0.025 \\
\hline
\end{tabular}

2 cases of trilateral RB had gene pathogenic variants; The proportion of $R B 1$ gene site pathogenic variant in children with family genetic risk factors was significantly higher than that in children without family genetic risk factors $(P=0.021$ ); there was also a difference between age factors and $R B 1$ gene pathogenic variants. Chai et al. found that $R B 1$-mutated patients presented with earlier age of diagnosis, with a significantly larger proportion of bilateral cases and secondary malignancies relative to those without RB1 mutations [24]. To some extent, our results were consistent with those of Chai et al. Therefore, it indicated that the pathogenic variant of the $R B 1$ gene was closely related to the onset characteristics. We also observed that the proportion of $R B 1$ gene pathogenic variant in children $>12$ months was higher than that in infants $(59.3 \%$ vs. $16.7 \%, P=0.034)$, which was different from previous literature reports and should be further analyzed of its pathogenesis, which might provide a basis for future diagnosis and treatment. Although the pathogenic variant rate of $R B 1$ gene in infant $R B$ was lower than that in $\mathrm{RB}$ children aged $>12$ months, the pathogenic variant rate of infants with bilateral RB was relatively high, and it was mainly inherited by species, from the results of our study. In our study, the cause of some unilateral $\mathrm{RB}$ in aged $>12$ months was related to $R B 1$ gene site mutation, although without family genetic factors, which might lead to tumor after the second hit of $\mathrm{RB}$ cells reported in previous reports $[1,5,6]$. The proportion of $R B 1$ gene pathogenic variant in
$\mathrm{RB}$ of bilateral/trilateral eyes was significantly increased in this group, including the older age group. $R B 1$ gene pathogenic variant was not limited to children with previous infantile onset, while familial genetic factors were not dominant in children over 12 months old. Therefore, we suggested that $R B 1$ gene pathogenic variant screening should be paid attention to in children with bilateral and/or trilateral RB under 5 years old, so as to do a good job in genetic counseling.

Identification of the $R B 1$ gene mutation and the genetic pattern was helpful to strengthen the clinical management of high-risk relatives [25]. RB gene mutation analysis can help nurses to improve the management of RB patients from different aspects, guide the screening plan for some high-risk relatives of $\mathrm{RB}$ patients with positive $R B 1$ mutation detection, and eliminate unnecessary screening for relatives without disease risk. From the analysis of $R B 1$ gene pathogenic variant types, frameshift pathogenic variants accounted for $57.9 \%(11 / 19)$, and base-missing/insertion missense pathogenic variants accounted for $42.1 \%(8 / 19)$. However, in nongermline inherence, base deletion/insertion accounts for $60 \%$, indicating that for genetic $R B 1$ frameshift pathogenic variants are mostly occurred to transmit genetic information, while for nongermline inheritance, due to tumor gene pathogenic variants mostly occurred, their own missense pathogenic variants become the main cause of disease. Due to the lack of RB protein, nonsense and frameshift mutations often lead to bilateral multifocal tumors [26]. In addition, our 
study found that 10 undefined pathogenic variant sites existed in 10 children, while it needs further verification to identify whether it is a clear harmful pathogenic variant. As we can see from Table 3, there is a heterozygous pathogenic variant at position c.2455C $>\mathrm{G}$ (p.L819V) in the proband (case 2) and his mother at the same time. Therefore, this pathogenic variant has not been reported, and it should be concerned. In case 8 of Table 3 , the child has a missense pathogenic variant in base deletion. Although the mother was not detected to have the $R B 1$ gene pathogenic variant, she still had a unilateral eye disease. Therefore, it should be noted that the genetic mutation of the offspring may be caused by genetically high-risk factors.

The treatment of RB should be based on the primary purpose of saving life, and a variety of treatment methods $[27,28]$ should be adapted. The current treatment regimens were based on a variety of different factors, such as clinical staging at initial diagnosis, intraocular staging, onset eyes, family genetics, and family decisions, including conservative treatment (intervention, glass group removal, laser, and freezing), surgical resection including eyeball removal and orbital content removal, and systemic chemotherapy and radiotherapy treatment. For distant metastasis, stem cell transplantation could also be used in children at this stage $[20,21,29]$. At present, the overall survival rate in developed countries and China has reached more than 90\%; however, the mortality rate in backward countries such as Kenya is still high $[1,3,6]$. This may cause by the lack of timely diagnosis and treatment.

Although the children in this group mainly developed RB in the intraocular period, only 2 cases were a special type of $\mathrm{RB}$ in the saddle area and the pituitary, while they still mainly in the D and E stages. Therefore, systemic chemotherapy combined with radiotherapy and sheath injection therapy was mainly used, and local treatment was combined in some children. Prognostic analysis suggests that the removal of the eyeball, the presence of recurrence, and the use of sheath injection therapy in children after posterior optic nerve invasion are all risk factors that affect the prognosis, regardless of whether there is a family history or genetic pathogenic variant. The above results showed that the prognosis of RB was affected by many factors, especially the treatment. Although the patients with $R B 1$ gene pathogenic variant are relatively complex, reasonable treatment can still achieve a better long-term survival. But our research is still insufficient, most of the patients in our clinic were sporadic cases, so our series included sporadic cases and a few familial cases. However, we believe that our findings may help to facilitate further largescale studies to investigate this correlation.

In conclusion, the mutation of the $R B 1$ gene and the presence of family genetic risk factor are closely related to the occurrence of RB disease, and the prognosis should also be combined with multiple factors such as patient's tolerance to the treatment, diagnosis, and treatment methods. Pathogenic variants of 10 nucleic acid sites without reported were found in this study, among which c.2455C $>$ G (p.L819V) was confirmed to have heterozygous mutations in a bilateral $\mathrm{RB}$ patient and his mother with unilateral RB. The proportion of pedigree inheritance of infantile retinoblastoma with bilateral disease was higher. There was a certain proportion of RB1 gene mutation in 3-5-year-old children with bilateral $\mathrm{RB}$, even if they had no family genetic history. Therefore, the detection of the absence or pathogenic variant of the $R B 1$ gene has positive significance for clinical diagnosis and treatment, and we suggest that the detection of $R B 1$ gene mutation should not only focus on infants but also on the phenotype of $R B 1$ gene mutation in children over 3 years old with bilateral eye disease.

\section{Data Availability}

The data in this manuscript are available from the corresponding author upon request.

\section{Ethical Approval}

Our study was approved by the Ethics Committee of Beijing Tongren Hospital, Capital Medical University.

\section{Consent}

Written informed consent of the legal guardian was obtained.

\section{Conflicts of Interest}

The authors declare that there were no conflicts of interest in the study.

\section{Authors' Contributions}

Yi Zhang and Yizhuo Wang contribute equally to the manuscript and thus share the first authorship.

\section{Acknowledgments}

This study was supported by the Beijing Municipal Administration of Hospitals Mission Plan (grant number DFL20180201 and DFL 20190201).

\section{References}

[1] H. Dimaras, K. Kimani, E. A. O. Dimba et al., "Retinoblastoma," Lancet, vol. 379, no. 9824, pp. 1436-1446, 2012.

[2] M. C. de Jong, W. A. Kors, P. de Graaf, J. A. Castelijns, T. Kivelä, and A. C. Moll, "Trilateral retinoblastoma: a systematic review and meta-analysis," The Lancet Oncology, vol. 15, no. 10, pp. 1157-1167, 2014.

[3] A. AlAli, S. Kletke, B. Gallie, and W. C. Lam, "Retinoblastoma for pediatric ophthalmologists," Asia-Pacific Journal of Ophthalmology, vol. 7, no. 3, pp. 160-168, 2018.

[4] T. Kivelä, "The epidemiological challenge of the most frequent eye cancer: retinoblastoma, an issue of birth and death," The British Journal of Ophthalmology, vol. 93, no. 9, pp. 1129$1131,2009$.

[5] S. E. Soliman, H. Racher, C. Zhang, H. MacDonald, and B. L. Gallie, "Genetics and molecular diagnostics in retinoblastoma-an update," Asia-Pacific Journal of Ophthalmology, vol. 6, no. 2, pp. 197-207, 2017. 
[6] H. Dimaras, T. W. Corson, D. Cobrinik et al., "Retinoblastoma," Nature Reviews Disease Primers, vol. 1, no. 1, p. 15021, 2015.

[7] H. Ishaq and B. C. Patel, Retinoblastoma, in StatPearls, StatPearls Publishing LLC., Treasure Island (FL), 2021.

[8] B. Desvoyes and C. Gutierrez, "Roles of plant retinoblastoma protein: cell cycle and beyond," The EMBO Journal, vol. 39, no. 19, article e105802, 2020.

[9] X. Lan, W. Xu, X. Tang et al., "Spectrum of RB1 germline mutations and clinical features in unrelated Chinese patients with retinoblastoma," Frontiers in Genetics, vol. 11, p. 142, 2020.

[10] A. Mokánszki, Y. C. Chang Chien, J. A. Mótyán et al., "Novel RB1 and MET gene mutations in a case with bilateral retinoblastoma followed by multiple metastatic osteosarcoma," Diagnostics, vol. 11, no. 1, p. 28, 2021.

[11] X. L. Xu, H. P. Singh, L. Wang et al., "Rb suppresses human cone-precursor-derived retinoblastoma tumours," Nature, vol. 514, no. 7522, pp. 385-388, 2014.

[12] A. M. Raizis, H. M. Racher, A. Foucal, H. Dimaras, B. L. Gallie, and P. M. George, "DNA hypermethylation/boundary control loss identified in retinoblastomas associated with genetic and epigenetic inactivation of theRB1gene promoter," Epigenetics, vol. 1, pp. 1-15, 2020.

[13] S. A. Henley, S. M. Francis, J. Demone, P. Ainsworth, and F. A. Dick, "A cancer derived mutation in the retinoblastoma gene with a distinct defect for LXCXE dependent interactions," Cancer Cell International, vol. 10, no. 1, p. 8, 2010.

[14] R. Kaewkhaw and D. Rojanaporn, "Retinoblastoma: etiology, modeling, and treatment," Cancers (Basel), vol. 12, no. 8, p. 2304, 2020.

[15] E. O. Berge, S. Knappskog, S. Geisler et al., "Identification and characterization of retinoblastoma gene mutations disturbing apoptosis in human breast cancers," Molecular Cancer, vol. 9, no. 1 , p. 173, 2010.

[16] K. Roelofs, F. Shaikh, W. Astle, B. L. Gallie, and S. E. Soliman, "Incidental neuroblastoma with bilateral retinoblastoma: what are the chances?," Ophthalmic Genetics, vol. 39, no. 3, pp. 410413, 2018.

[17] J. Shields and C. Shields, "Retinoblastoma: diagnostic approaches," in In Atlas of Intraocular Tumors, Lippinoctt, Wolters Kluwer, Philadelphia, 3rd ed edition, 2016.

[18] G. Chantada, F. Doz, C. B. G. Antoneli et al., "A proposal for an international retinoblastoma staging system," Pediatric Blood \& Cancer, vol. 47, no. 6, pp. 801-805, 2006.

[19] C. L. Shields, A. Mashayekhi, A. K. Au et al., "The international classification of retinoblastoma predicts chemoreduction success," Ophthalmology, vol. 113, no. 12, pp. 2276-2280, 2006.

[20] R. Rao and S. G. Honavar, "Retinoblastoma," Indian Journal of Pediatrics, vol. 84, no. 12, pp. 937-944, 2017.

[21] R. J. Bowman, M. Mafwiri, P. Luthert, J. Luande, and M. Wood, "Outcome of retinoblastoma in East Africa," Pediatric Blood \& Cancer, vol. 50, no. 1, pp. 160-162, 2008.

[22] R. Leclerc and J. Olin, "An overview of retinoblastoma and enucleation in pediatric patients," AORN Journal, vol. 111, no. 1, pp. 69-79, 2020.

[23] P. K. Shah, S. Sripriya, V. Narendran, and A. J. Pandian, "Prenatal genetic diagnosis of retinoblastoma and report of RB1 gene mutation from India," Ophthalmic Genetics, vol. 37, no. 4, pp. 430-433, 2016.
[24] P. Chai, Y. Luo, J. Yu et al., "Clinical characteristics and germline mutation spectrum of RB1 in Chinese patients with retinoblastoma: a dual-center study of 145 patients," Experimental Eye Research, vol. 205, p. 108456, 2021.

[25] Z. Zhang, Y. S. Xiao, R. Shen et al., "Next generation sequencing of RB1gene for the molecular diagnosis of ethnic minority with retinoblastoma in Yunnan," BMC Medical Genetics, vol. 21, no. 1, p. 230, 2020.

[26] M. K. Mohd Khalid, Y. Yakob, R. Md Yasin et al., "Spectrum of germ-line RB1 gene mutations in Malaysian patients with retinoblastoma," Molecular Vision, vol. 21, pp. 1185-1190, 2015.

[27] M. Amin, S. Edge, and F. Greene, "Retinoblastoma," in AJCC Cancer Staging Manual, Springer, New York, NY, 8th ed edition, 2017.

[28] A. Singh, L. Murphee, and B. Damato, "Orbital retinoblastoma," in Clinical Ophthalmic Oncology-Retinoblastoma, pp. 185-194, Springer, NY, 2nd ed edition, 2015.

[29] J. Chen, C. Rodríguez, R. Monestel, and A. Zúñiga, "Clinical, epidemiological, and therapeutic profile of patients with a retinoblastoma diagnosis experience in the Costa Rica National Children's Hospital Oncology Unit from January 2009 to December 2015," Ecancermedicalscience, vol. 11, p. 754, 2017. 